Revista de Matemática: Teoría y Aplicaciones 2010 17(1) : 81-101

CIMPA - UCR ISSN: 1409-2433

\title{
JUEGOS REPETITIVOS Y SISTEMAS DINÁMICOS EN EL MERCADO DEL PETRÓLEO
}

\section{REPEATING GAMES AND DYNAMICAL SYSTEMS IN OIL MARKET}

\author{
Osvaldo Acuña Ortega* Fernán Ulate Montero**
}

Received: 30 Nov 2008; Revised: 1 Oct 2009; Accepted: 21 Oct 2009

Palabras clave: Economía matemática, precios del petróleo, modelos matemáticos.

Keywords: Mathematical Economics, oil prices, mathematical models. Mathematics Subject Classification: 91B55.

${ }^{*}$ CIMPA-Escuela de Matemática, Universidad de Costa Rica, 2060 San José, Costa Rica. E-Mail: MaritzaAvendanoRivera@hotmail.es

**Escuela de Economía, Universidad de Costa Rica, 2060 San José, Costa Rica. 


\begin{abstract}
Se utiliza la teoría moderna de juegos repetitivos en un modelo que ayuda a comprender un mercado como el de la OPEP. Se estudia, además, un sistema dinámico del tipo Lotka-Volterra, y se analiza el modelo.
\end{abstract}

\title{
Resumen
}

We use the modern theory of repetitive games in a model that help understand a market with a cartel like OPEP. We also study a dynamical system Lotka-Volterra type, and we analyze the dynamic behavior of the model.

\section{Análisis teórico}

Primero que nada, se presenta un modelo sencillo, que puede ayudarnos a comprender mejor el mercado actual del petróleo. Suponemos que existe una demanda del tipo lineal mundial por el producto y que hay dos tipos de países o regiones que ofrecen el producto:

i. Aquellos no afiliados a la OPEP, y que prácticamente ponen en el mercado todo lo que su infraestructura productiva permite.

ii. Aquellos afiliados a un cartel como la OPEP, que pretende funcionar como un monopolio, y que se enfrenta a la demanda residual, la cual se obtiene restando a la demanda mundial del petróleo la oferta de los países no miembros de la OPEP.

Si el cartel maximiza monopólicamente la ganancia de sus miembros, tendríamos una situación clara y muy sencilla de comportamiento económico. El problema es que sus miembros están valorando constantemente la capacidad de hacer fraude. Dejamos para investigación futura las necesidades económico-políticas y sociales de los gobiernos de cada país, que nos llevarían a un comportamiento complejo, en donde una gran cantidad de variables no económicas deben ser incluidas, y nos concentramos en el aspecto económico-racional de si es mejor o no hacer fraude y estudiamos algunos aspectos importantes que afectan dicha decisión. Se hacen cálculos económico-matemáticos, y se resumen en un cuadro que expondremos en el apartado siguiente.

Esencialmente, hacer fraude tiene dos consecuencias:

i. Una ganancia en el presente. 
ii. Una pérdida en el futuro, porque suponemos que las otras empresas van a responder al fraude haciendo lo mismo, y como consecuencia el cartel deja de funcionar como tal, llevándonos, suponemos, a un equilibrio de Nash.

De este punto de vista del modelo en adelante nos dedicaremos a estudiar cómo los cambios en la demanda presente y futura afectan el modelo.

Nos concentraremos en dos tipos de cambios:

i. La demanda residual presente.

ii. La demanda residual futura, en la que suponemos, como una primera aproximación, que la demanda residual cambia en el período siguiente y se mantiene igual por el resto de períodos.

- Si la demanda residual aumenta y los miembros de la OPEP consideran que la demanda residual futura va a ser la misma que se tenía anteriormente, entonces el castigo por hacer fraude sería el mismo, pero la ganancia sería mayor, con lo que, potencialmente, se estimula la oferta del producto, haciendo que el aumento de precio, resultado de una mayor demanda residual transitoria, se compense o disminuya. Este fenómeno se podría dar, ya sea porque la demanda mundial aumenta transitoriamente, o porque transitoriamente hay un problema en la extracción, transporte infraestructura o producción de algún país o región no miembro de la OPEP.

- Si la demanda residual presente permanece constante, y los miembros de la OPEP consideran que la demanda residual futura va a aumentar, ya sea porque la demanda mundial total aumenta (mayor desarrollo e industrialización de países grandes como China y la India), o porque la producción de los países o regiones no miembros de la OPEP va a disminuir, entonces las ganancia de hacer fraude sería la misma, pero la pérdida aumentaría desestimulando el fraude, aumentando así la lealdad de los miembros de la OPEP, y potencialmente disminuyendo la oferta y aumentando los precios, reforzando este hecho en el futuro el aumento de los precios, producto de una mayor demanda residual en el futuro.

Si por el contario, tenemos el caso en el que los miembros de la OPEP consideran que la demanda residual futura va a disminuir, ya sea porque los países no miembros de la OPEP aumentarían la producción (nuevos descubrimientos petroleros, regiones por explotar, mejor tecnología, etc.) o que la demanda futura mundial va a 
disminuir (por ejemplo, nuevas tecnologías que efectivamente sustituyan la demanda de petróleo, cambios culturales de consumo, una fuerte recesión mundial, etc.) entonces, la pérdida o costo futuro por hacer fraude disminuiría, estimulando la producción por parte de los miembros de la OPEP, y ventas a escondidas de petróleo, con lo que la oferta aumentaría, disminuyendo los precios aún más de lo que la demanda por si sola los habría disminuido.

En resumen, el modelo expone un comportamiento en el mercado de petróleo, que hace mucho más volátil y sorpresivo el comportamiento de los precios.

En el apartado 3 de este trabajo, expondremos los resultados de un estudio empírico sencillo y preliminar que debe mejorarse con técnicas modernas de econometría, y que efectivamente nos brinda una idea de la volatilidad de este mercado. También se analiza el comportamiento dinámico del modelo Lotka-Volterra que se expone en la última parte del artículo.

Esencialmente, las variables precio y cantidad no tienden "suavemente" al equilibrio, sino que siguen una trayectoria espiral; si la curva de demanda cambiara entonces tendríamos toda una nueva espiral definida por la nueva curva de demanda con las fluctuaciones y volatilidad propias de la nueva situación. Así tenemos un modelo que describe la gran volatilidad que en la realidad se encuentra en el mercado del petróleo.

\section{Lealtad y fraude en el cartel del petróleo. Desarrollo matemático}

En este apartado estudiaremos un modelo simplificado que nos pueda ayudar a comprender cómo aumentos en la demanda esperada de un bien como el petróleo pueden producir disminuciones en la oferta de ese bien.

Suponemos que en un mercado existen $m+n$ empresas (o regiones) o países de los cuales $m$ producen toda la cantidad del bien que su infraestructura productiva permite y los $n$ restantes podrían potencialmente integrar un cartel. Cada uno de los $m$ países produce una cantidad $k_{j}$ del bien y en total producen una cantidad $K$.

$$
K=\sum_{j=1}^{m} k_{j} \quad k_{j} \in \mathbb{R}^{+}=\{x \in \mathbb{R} / x \geq 0\}
$$


Sea la demanda del bien

$$
q=\alpha-\beta p \quad \alpha, \beta \in \mathbb{R}^{++}=\{x \in \mathbb{R} / x>0\}
$$

donde $q=$ cantidad del bien, $p=$ precio del bien, $p, q \in \mathbb{R}^{+}$.

Las $n$ empresas o países restantes (que son los que más nos interesan) enfrentan la demanda residual y pueden:

i. organizarse en cartel (monopolio), y ahí pueden decidir si hacer fraude o no,

ii. organizarse en equilibrio Cournot (equilibrio de Nash).

La demanda residual será

$$
q=\alpha-\beta p-K
$$

donde suponemos $\alpha-K>0$.

Entonces podemos reescribir (3) así

$$
p=a-b q, \text { donde } a=\frac{\alpha-K}{\beta} ; \quad b=\frac{1}{\beta} ; \quad a, b \text { parámetros. }
$$

Suponemos que cada empresa o país tiene costos de producción promedio constantes e idénticos

$$
C_{i}=c q_{i}
$$

donde $C_{i}$ es el costo de producción total del país o empresa, $i(i=1, \ldots n)$, $q_{i}=$ producción de empresa $i(i=\ldots n), c \in \mathbb{R}^{++}, c<a, q_{i} \in \mathbb{R}^{+}$; $q=\sum_{i=1}^{n} q_{i}$

Si las empresas se organizan en un Monopolio (Cartel) y se respetan las coutas de producción en partes iguales entonces se tendrá que

$$
q_{i}=\frac{q_{m}}{n}
$$

donde $q_{m}$ soluciona el siguiente problema de maximización de ganancias

$$
\max _{q}(a-b q) q-c q
$$

Las condiciones de primer orden son necesarias y suficientes para un máximo puesto que la función es cóncava. 
En la tabla 1 se resumen los resultados de este proceso de organización donde $p_{m}=$ precio de monopolio, $q_{m}=$ cantidad producida por el monopolio, $q_{m i}=$ cantidad producida por país o empresa del monopolio, $\pi_{m i}=$ ganancias de cada país o empresa del monopolio.

En el equilibrio de Cournot cada empresa o país maximiza sus ganancias tomando como un dato la producción de los demás, al actuar todos de la misma forma el resultado que se alcanzaría en equilibrio es un "Equilibrio de Nash".

Así tenemos para cada empresa o país

$$
\max _{q_{i}}\left(a-b\left(\sum_{j \neq i} q_{j}\right)-b q_{i}-c\right) q_{i}, \quad(i=1, \ldots n)
$$

Como la función es cóncava en $q_{i}$ las condiciones de primer orden son necesarias y suficientes para un máximo.

Tenemos así un sistema de $n$ ecuaciones de (7) y de $n$ incógnitas (cada una de las $\left.q_{i}\right)$.

\begin{tabular}{l|cccc}
\hline \hline Monopolio & $p_{m}$ & $q_{m}$ & $q_{m i}$ & $\pi_{m i}$ \\
& $\frac{a+c}{2}$ & $\frac{a-c}{2 b}$ & $\frac{a-c}{2 b n}$ & $\frac{(a-c)^{2}}{4 b n}$ \\
\hline Equilibrio de Nash & $p_{c}$ & $q_{c}$ & $q_{c i}$ & $\pi_{c i}$ \\
(Cournot) & $\frac{a+n c}{n+1}$ & $\frac{n(a-c)}{b(n+1)}$ & $\frac{a-c}{b(n+1)}$ & $\frac{(a-c)^{2}}{b(n+1)^{2}}$ \\
\hline Fraude & $p_{f}$ & $q_{f}$ & $q_{f o}$ & $\pi_{f o}$ \\
& $\frac{n a+3 n c+a-c}{4 n}$ & $\frac{(a-c)(3 n-1)}{4 b n}$ & $\frac{(n+1)(a-c)}{4 b n}$ & $\frac{(n+1)^{2}(a-c)^{2}}{16 b n^{2}}$ \\
\hline \hline
\end{tabular}

Tabla 1: Resultados del equilibrio.

Los resultados de este equilibrio se exponen en la tabla 1, donde:

- $q_{c i}=$ cantidad que produce cada empresa o país en equilibrio de Cournot.

- $q_{c}=$ total de producción en equilibrio de Cournot por las $n$ empresas o países

- $p_{c}=$ precio en equilibrio de Cournot.

- $\pi_{c i}=$ ganancia de cada empresa o país en equilibrio de Cournot. 
Ahora bien, primero partamos de la idea que al inicio del análisis las empresas o países se encuentran funcionando en un cartel (o monopolio).

Supongamos que una de las empresas (llámela o) está considerando hacer fraude y trata de maximizar sus ganacias sujeto a que las demás empresas $(i \neq o)$ mantienen la producción o cuota del cartel o monopolio.

Así tenemos para la empresa $o$

$$
\max _{q_{0}}\left(a-b(n-1) \bar{q}_{m i}-b q_{0}-c\right) q_{0}
$$

donde $\bar{q}_{m i}$ es el valor correspondiente al monopolio en la tabla 1, la cual sustituimos y obtenemos

$$
\max _{q_{0}}\left(a-\left(\frac{(n-1)(a-c)}{2 n}\right)-b q_{0}-c\right) q_{0}
$$

De nuevo la concavidad nos asegura que las condiciones de primer orden son necesarias y suficientes para el máximo.

La tabla 1 nos daría los resultados correspondientes a esta situación, donde

- $\pi_{f o}=$ ganancia de empresa o país que haría fraude.

- $q_{f o}=$ producción de empresa o país que haría fraude.

- $q_{f}=$ cantidad producida por las $n$ empresas.

- $p_{f}=$ precio de mercado.

Suponemos que la empresa o país que hace fraude sabe que si hace fraude en el período siguiente las otras empresas del cartel imitarán su comportamiento y la nueva situación del mercado sería un equilibrio de Cournot. Entonces es claro que si hace fraude en el presente gana $\pi_{f o}>\pi_{m o}$ pero en el futuro pierde, puesto que $\pi_{c o}<\pi_{m o}$.

La empresa o país tiene que tomar una decisión de escogencia intertemporal y tiene que tener un parámetro $(\delta>0)$ que sirve para traer a valor presente las pérdidas o ganancias futuras.

Sea $F$ : la ganancia total neta de hacer fraude sobre la situación de monopolio. Así

$$
F=\left(\pi_{f o}-\pi_{m o}\right)+\frac{\pi_{c o}-\pi_{m o}}{(1+\delta)}+\frac{\pi_{c o}-\pi_{m o}}{(1+\delta)^{2}}+\cdots
$$

Como $\delta>0$ la serie converge y tenemos

$$
F=\left(\pi_{f o}-\pi_{m o}\right)+\frac{1}{\delta}\left(\pi_{c o}-\pi_{m o}\right) .
$$


El primer sumando es positivo, el segundo sumando es negativo, la empresa hará fraude si $F>0$.

Ahora bien, ¿cómo cambia la ganancia total neta de hacer fraude si la demanda del bien aumenta $d a>0$ ?, el lector podrá comprobar que $\frac{d F}{d a}$ es una expresión compleja que tiene una parte positiva y otra negativa y es indefinita puesto que depende de $\delta$.

Para analizar la situación que se nos presenta vamos a suponer que hay dos tipos de demanda, una para el presente y otra para los períodos futuros (igual para todos los períodos futuros) que pueden variar independientemente una de la otra. O sea, la demanda presente puede aumentar o disminuir o quedar igual y la futura independientemente puede quedar igual o variar.

Así tenemos

- $d^{0}=$ demanda presente $(t=0)$,

- $d^{1}=$ demanda futura $(t=1,2,3)$,

- $d^{0}=a^{0}-b p$

- $d^{1}=a^{1}-b p$

y tenemos para la empresa $o$

$\pi_{m o}^{0}=$ ganancia de monopolio en presente,

$\pi_{c o}^{0}=$ ganancia de Cournot en presente,

$\pi_{f o}^{0}=$ ganancia de hacer fraude en presente,

$\pi_{m o}^{1}=$ ganancia de monopolio en futuro,

$\pi_{c o}^{1}=$ ganancia de Cournot en futuro,

$$
a^{0} \geqq a^{1} \quad \text { o } \quad a^{1} \geqq a^{0} .
$$

Supongamos que la empresa "o" que es parte del cartel que actúa monopólicamente en el presente y está valorando si hace fraude o no en el presente.

La ganancia neta en el presente de hacer fraude en el presente es

$$
W^{0}=\pi_{f o}^{0}-\pi_{m o}^{0}
$$


La ganancia neta en el futuro de hacer fraude en el presente es

$$
W^{1}=\pi_{c o}^{1}-\pi_{m o}^{1}
$$

La ganancia total neta (presente y futura) de hacer fraude sería

$$
F=W^{0}+\frac{W^{1}}{1+\delta}+\frac{W^{1}}{(1+\delta)^{2}}+\frac{W^{1}}{(1+\delta)^{3}}+\ldots
$$

donde $\delta$ es el factor de preferencia temporal (14) se podría expresar así

$$
F=W^{0}+\frac{1}{\delta} W^{1}
$$

donde

$$
\begin{gathered}
W^{0}=\frac{\left(a^{0}-c\right)^{2}(n-1)^{2}}{16 b n^{2}}>0 \\
W^{1}=\frac{-\left(a^{1}-c\right)^{2}(n-1)^{2}}{4 b n(n+1)^{2}}<0
\end{gathered}
$$

(pérdida porque los otros miembros responderían haciendo que el cartel no funcione en el futuro). Tenemos $F=F\left(a^{0}, a^{1}\right)$, puesto que $a^{0}$ y $a^{1}$ son variables. Ahora tenemos, ante cambios en $a^{0}$ y $a^{1}$

$$
\begin{gathered}
\frac{\partial F}{\partial a^{0}}=\frac{2\left(a^{0}-c\right)(n-1)^{2}}{16 b n^{2}}>0 \\
\frac{\partial F}{\partial a^{1}}=\frac{-2\left(a^{1}-c\right)(n-1)^{2}}{4 b n(n+1)^{2} \delta}<0 .
\end{gathered}
$$

O sea, si la demanda residual presente aumenta y los miembros del cartel estiman que dicho aumento es transitorio, entonces la ganancia total neta de hacer fraude aumentaría y este efecto estimularía la producción más allá de las cuotas del cartel monopólico.

Se aprovecharía así la época de vacas gordas que sería transitoria (ver por ejemplo Rotemberg y Saloner).

Pero si es la demanda residual futura la que aumenta y la presente no cambia o cambia poco, entonces este efecto disminuye la ganancia neta de hacer fraude, estimulando la lealtad entre los miembros del cartel, facilitando así la disminución de la oferta por parte del cartel, potenciando un aumento en los precios.

Si los países de la OPEP estiman que de la misma forma, la demanda global futura disminuye o aumenta la oferta futura de los países petroleros que no estén en la OPEP; en el presente entonces disminuye la lealtad de 
los países de la OPEP al cartel aumentando potencialmente la oferta y la volatilidad en los precios.

Es importante comprender que los miembros de un cartel como la OPEP, son países cuyos gobiernos tienen una serie de compromisos socialeseconómicos y políticos con los pueblos. Esto hace que de alguna forma, el factor de preferencia temporal se afecte con los precios aumentando aún más la volatilidad del modelo, siendo este tema político-cultural un campo interesante para una investigación futura.

\section{Presentación del sistema dinámico}

En este apartado, primero mostaremos un sistema dinámico tipo LotkaVolterra, del cual se hizo una estimación preliminar empírica y posteriormente analizaremos la trayectoria dinámica de dicho sistema.

La estimación se hace utilizando la metodología de cuadrados mínimos ordinarios, que, desde luego, puede mejorarse con técnicas más avanzadas de econometría.

Tenemos

$$
\begin{gathered}
\frac{p_{f}-p_{i}}{p_{i}}=\alpha+\beta p_{i}+\gamma q_{i}+\mu \\
\frac{q_{f}-q_{i}}{q_{i}}=\omega+\epsilon p_{i}+\mu
\end{gathered}
$$

donde:

$p_{i}=$ precio mundial del petróleo en el mes $i$, se utilizó como referencia el precio del West Texas Crude, deflatado con el índice de precios al consumidor de Estados Unidos (dólares constantes).

$p_{f}=$ precio mundial del petróleo un año después del período $i$.

$q_{i}=$ oferta mundial del petróleo em período $i$ (promedio diario en miles de barriles).

$q_{f}=$ oferta mundial del petróleo un año después del período $i$.

(Datos del año 1946 al año 2006, inclusive). $\alpha, \beta, \gamma, \epsilon, \omega$ parámetros a estimar, con el resultado siguiente (debajo de cada parámetro se pone el nivel de significancia de éste y a la par de la ecuación se escribe el resultado para el estadístico $F$ y su nivel de significancia).

$$
\frac{p_{f}-p_{i}}{p_{i}}=-.334 \quad-\quad .001 \quad p_{i}+.003 \quad q_{i}+\mu
$$


(F: $7.369(.001))$

$\frac{q_{f}-q_{i}}{q_{i}}=\underset{(.002)}{.014}-\begin{array}{ccc}.001 & p_{i}++\mu\end{array}$

(F: $7.886(.005))$

El resultado muestra que los $F$ son muy significantes para las dos ecuaciones, además muestra que las $t$ - estadística de cada parámetro son significantes, con excepción del parámetro $\alpha$.

Esta estimación es útil para estudiar las ecuaciones de Lotka-Volterra que utiliza ecuaciones diferenciales, y obtener así valores para el siguiente sistema dinámico sencillo.

$$
\left\{\begin{array}{c}
\frac{d p}{d t}=(\alpha+\beta p+\gamma q) p \\
\frac{d q}{d t}=(\omega+\epsilon p) q
\end{array}\right.
$$

con $\alpha<0, \beta<0, \gamma>0, \omega>0, \epsilon<0$.

Estudiaremos a continuación, el comportamiento del sistema dinámico (18).

$$
\left\{\begin{array}{c}
\frac{d p}{d t}=(-a-b p+c q) p \\
\frac{d q}{d t}=(w-e p) q
\end{array}\right.
$$

con $a, b, c, w, e>0$.

Podemos encontrar tres soluciones de esta ecuación diferencial:

i. $p_{1}(t)=0, q_{1}(t)=0, \forall t \in \mathbb{R}$

ii. $p_{2}(t)=0, q_{2}(t)=q_{2}(0) e^{w t}, \forall t \in \mathbb{R}, q_{2}(0)>0$

iii. $p_{3}(t)=\frac{a p_{3}(0)}{\left(a+b p_{3}(0)\right) \mathbf{e}^{a t}-b p_{3}(0)}, \quad q_{3}(t)=0, p_{3}(0)>0$

$\forall t>t_{0}$ donde $t_{0}<0$ tal que $a+b p_{3}(0) \mathbf{e}^{a t}-b p_{3}(0)=0$

Las órbitas de cada una de las soluciones son los conjuntos

$$
\mathfrak{L}_{i}=\left\{\left(p_{i}(t), q_{i}(t)\right) / t \in\right] \tilde{t_{0}},+\infty[\} \quad \text { con } i=1,2,3 .
$$

y $\tilde{t_{0}}=-\infty$ si $i=1,2, \tilde{t_{0}}=t_{0}$ si $i=3$.

Se representan en $\mathbb{R}^{2}$ como se muestra en la Figura 1. 


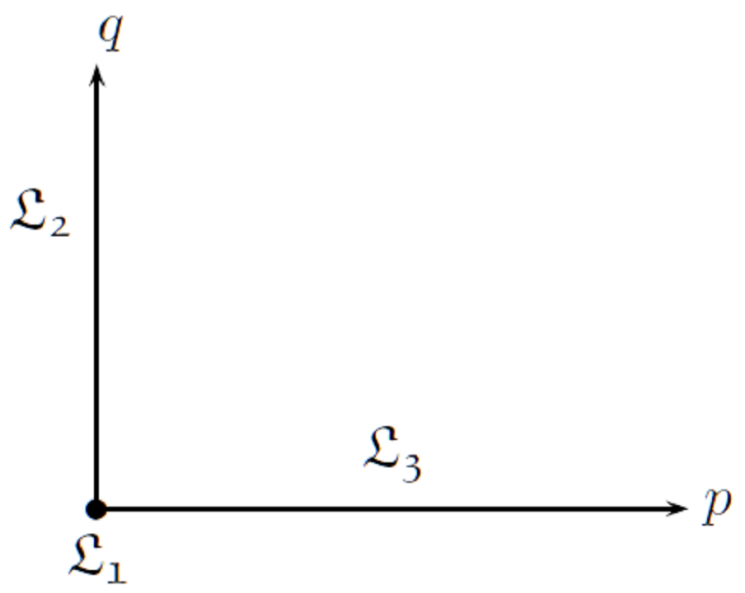

Figura 1: órbitas de la ecuación diferencial (19).

Definición 3.1 Sea $\dot{x}=f(x)$ una ecuación diferencial autónoma, donde $f(x)$ está definida en un conjunto abierto $D$ de $\mathbb{R}^{n}$. Un subconjunto $F$ de $D$ se dice ser invariante si para cada solución $x(t), t \in I$ ( $I$ un intervalo abierto) tal que existe $t_{0} \in I$ con $x\left(t_{0}\right) \in F$, entonces $\forall t \in I, x(t) \in F$.

Nota 3.1 Si $\mathbb{R}_{+}^{2}=\left\{(p, q) \in \mathbb{R}^{2} / p, q \geq 0\right\}$, entonces la frontera de $\mathbb{R}_{+}^{2} y$ el interior de $\mathbb{R}_{+}^{2}=\left\{(p, q) \in \mathbb{R}^{2} / p>0, q>0\right\}$ son conjuntos invariantes para la ecuación diferencial (19).

Probemos primero que la frontera de $\mathbb{R}_{+}^{2}$ es invariante. Sea $(p(t), q(t))$, $t \in I$ ( $I$ un intervalo abierto) una solución de la ecuación diferencial (19) tal que existe $t_{0} \in I$ con $\left(p\left(t_{0}\right), q\left(t_{0}\right)\right)$ en la frontera de $\mathbb{R}_{+}^{2}$. Podemos suponer que $t_{0}=0$, sin pérdida de generalidad reemplazando $(p(t), q(t))$ por $\left(p\left(t+t_{0}\right), q\left(t+t_{0}\right)\right)$ e $I$ por $I-t_{0}$. Entonces existe $i \in\{1,2,3\}$ tal que $(p(0), q(0)) \in \mathfrak{L}_{i}$. Por otro lado, $\left(p_{i}(t), q_{i}(t)\right)$, con $\left.t \in\right] \tilde{t_{0}},+\infty[$ es solución de la ecuación diferencial (19), con $p_{i}(0)=p(0), q_{i}(0)=q(0)$. El conjunto

$$
\{t \in] \tilde{t_{0}},+\infty\left[\cap I /\left(p_{i}(t), q_{i}(t)\right)=(p(t), q(t))\right\}
$$

es no vacío y cerrado en $] \widetilde{t_{0}},+\infty[\cap I$. Por el teorema de unicidad de soluciones para las ecuaciones diferenciales, sabemos que éste conjunto es abierto relativo de $] \tilde{t_{0}},+\infty[\cap I$ y como $] \widetilde{t_{0}},+\infty[\cap I$ es conexo, entonces se tiene que

$$
\left.\{t \in] \tilde{t_{0}},+\infty\left[\cap I /\left(p_{i}(t), q_{i}(t)\right)=(p(t), q(t))\right\}=\right] \tilde{t_{0}},+\infty[\cap I .
$$


Por otro lado si $1 \leq i \leq 2$ se tiene $\tilde{t_{0}}=-\infty$ y $] \tilde{t_{0}},+\infty[\cap I=I$, si $i=3$, $\tilde{t_{0}}=t_{0} \mathrm{y} \lim _{t \rightarrow t_{0}^{+}} p_{3}(t)=+\infty$, si $t_{0} \in I$, entonces $p_{3}(t)=p(t) \forall t>t_{0}$ y así se tiene que

$$
\begin{gathered}
+\infty=\lim _{t \rightarrow t_{0}^{+}} p_{3}(t)=\lim _{t \rightarrow t_{0}^{+}} p(t)=p\left(t_{0}\right) \\
\therefore p\left(t_{0}\right)=+\infty
\end{gathered}
$$

pero $p\left(t_{0}\right) \in \mathbb{R}$; lo cual es una contradicción y entonces debe tenerse que $t_{0} \notin I$, es decir $\tilde{t_{0}} \notin I$ y entonces $] \tilde{t_{0}},+\infty[\cap I=I$.

Así obtenemos que $\forall t \in I,\left(p_{i}(t), q_{i}(t)\right)=(p(t), q(t))$, lo que implica que $\forall t \in I,(p(t), q(t))$ pertenece a la frontera de $\mathbb{R}_{+}^{2}$.

Probemos ahora que el conjunto int $\left(\mathbb{R}_{+}^{2}\right)$ es invariante. Sea $(p(t), q(t))$, $t \in I$ ( $I$ intervalo abierto) una solución de (19) tal que existe $t^{\prime} \in I$ con $p\left(t^{\prime}\right), q\left(t^{\prime}\right)>0$. Probemos primero que $\{(p(t), q(t)) / t \in I\}$ no contiene puntos de la frontera de $\mathbb{R}_{+}^{2}$. Suponga por contradicción que existe $T \in I$ tal que $(p(T), q(T))$ pertenece a la frontera de $\mathbb{R}_{+}^{2}$. Sea $(\tilde{p}(t), \tilde{q}(t))=(p(t+$ $T), q(t+T)$ ) con $t \in I-T$ esto define una solución de la ecuación diferencial (19) tal que $(\tilde{p}(0), \tilde{q}(0))=(p(T), q(T))$ pertenece a la frontera de $\mathbb{R}_{+}^{2}$ y por la primera parte de ésta argumentación se tiene que $\left(p\left(t^{\prime}\right), q\left(t^{\prime}\right)\right)=\left(p\left(t^{\prime}-\right.\right.$ $T), q\left(t^{\prime}-T\right)$ ) pertenece a la frontera de $\mathbb{R}_{+}^{2}$, lo cual es una contradicción ya que $p\left(t^{\prime}\right), q\left(t^{\prime}\right)>0$. Por lo tanto, $\forall t \in I(p(t), q(t))$ no está en la frontera de $\mathbb{R}_{+}^{2}$. Como $p\left(t^{\prime}\right), q\left(t^{\prime}\right)>0$, no pueden existir puntos $(p(t), q(t))$ con $t \in I$ fuera de $\mathbb{R}_{+}^{2}$ ya que por la continuidad de $p(t), q(t)$ existirían puntos del conjunto $\{(p(t), q(t)) / t \in I\}$ en la frontera de $\mathbb{R}_{+}^{2}$, situación que no puede darse. Por lo tanto, $p(t), q(t)>0, \forall t \in I$.

Sólo estaremos interesados en soluciones de la ecuación diferencial

$$
\left\{\begin{array}{c}
\frac{d p}{d t}=(-a-b p+c q) p \\
\frac{d q}{d t}=(w-e p) q
\end{array}\right.
$$

con $a, b, c, w, e>0$, para $(p, q) \in \operatorname{int}\left(\mathbb{R}_{+}^{2}\right)$.

El conjunto de puntos $(p, q)$ donde $\dot{p}=0$ es llamado la $p$-isoclina y es donde el campo vectorial de la ecuación diferencial (19) es vertical. Este conjunto es $\{(p, q) /-b p+c q=a\}$. Similarmente el conjunto de las $q$-isoclinas es donde $\dot{q}=0$ y es el conjunto $\{(p, q) / e p=w\}$ y es donde el campo vectorial es horizontal. Ambos conjuntos se cortan en un único punto $F=(\bar{p}, \bar{q})$ en el $\operatorname{int}\left(\mathbb{R}_{+}^{2}\right)$ y se tiene que $F=(\bar{p}, \bar{q})=\left(\frac{w}{e}, \frac{b}{c}\left(\frac{a}{b}+\frac{w}{e}\right)\right)$. 


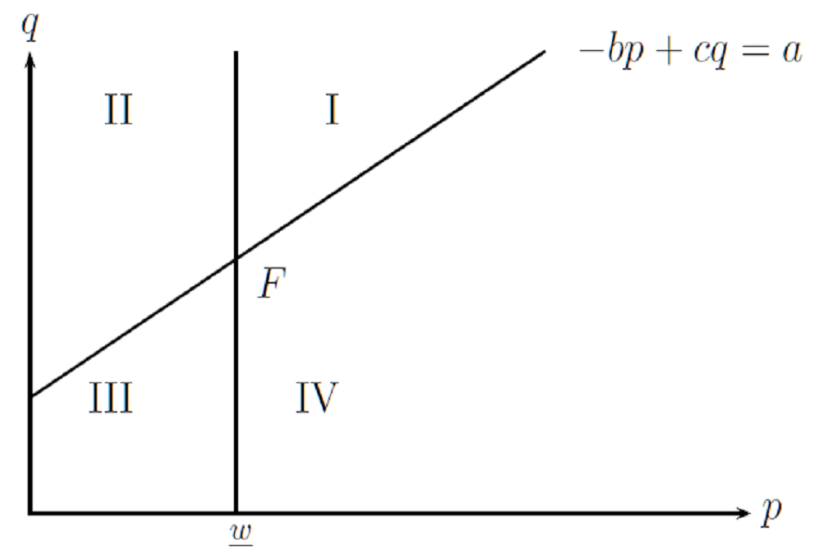

Figura 2: Regiones divididas por las isoclinas.

En este caso las isoclinas $e p=w \mathrm{y}-b p+c q=a$ dividen el interior de $\mathbb{R}_{+}^{2}$ en cuatro regiones I, II, III, IV, como se ve en la Figure 2.

Como $(\bar{p}, \bar{q})$ resuelve el sistema

$$
\left\{\begin{array}{c}
(-a-b p+c q) p=0 \\
(w-e p) q=0
\end{array}\right.
$$

entonces $(p(t), q(t))=(\bar{p}, \bar{q}) \forall t \in \mathbb{R}$, es una solución de la ecuación diferencial (19). En este caso la órbita de esta solución es $\{(\bar{p}, \bar{q})\}$, que es llamado punto de descanso de la ecuación diferencial $(19)$. $(\bar{p}, \bar{q})$ es el único punto de descanso de esta ecuación diferencial en $\operatorname{int}\left(\mathbb{R}_{+}^{2}\right)$.

Estudiaremos los signos de $\dot{p}, \dot{q}$ en las regiones I, II, III, IV.

$$
\dot{p}>0 \Longleftrightarrow-a-b p+c q>0 \Longleftrightarrow \frac{a+b p}{c}<q
$$

y entonces se tiene que $\dot{p}>0$ en I, II y $\dot{p}<0$ en III, IV.

Por otro lado,

$$
\dot{q}>0 \Longleftrightarrow w-e p>0 \Longleftrightarrow p<\frac{w}{e} ;
$$

entonces $\dot{q}<0$ en II, III y $\dot{q}<0$ en I, IV.

Las consideraciones de signo anteriores no son suficientes para especificar las órbitas de las soluciones de la ecuación diferencial (19), por lo tanto usaremos un poco de teoría de $\omega$-límites y funciones de Lyapunov. 
Definición 3.2 Sea $\dot{x}=f(x)$ un sistema autónomo en una región de $\mathbb{R}^{n}$ y sea $x(t)$ una solución del sistema definida para todo $t \geq 0$ tal que $x(0)=x$. El $\omega$-límite de $x$ es el siguiente conjunto

$\omega(x)=\left\{y \in \mathbb{R}^{n} / x\left(t_{k}\right) \rightarrow y\right.$ para alguna secuencia $\left(t_{k}\right)$ tq $\lim _{k \rightarrow+\infty} t_{k}=\infty$.

Nota 3.2 Podemos definir de manera análoga el conjunto $\alpha$-límite de $x$ como

$\alpha(x)=\left\{y \in \mathbb{R}^{n} / x\left(t_{k}\right) \rightarrow y\right.$ para alguna secuencia $\left(t_{k}\right) t q \lim _{k \rightarrow+\infty} t_{k}=-\infty$.

\section{Observación 4.1}

a. Si $\{x(t) / t \geq 0\}$ está contenido en un conjunto compacto, entonces $\omega(x) \neq \emptyset$.

b. Si $z \in\{x(t) / t \geq 0\}$ entonces $\omega(z)=\omega(x)$.

c. $\omega(x)=\bigcap_{t \geq 0} \overline{\{x(s) / s \geq t\}}$; en particular $\omega(x)$ es un conjunto cerrado de $\mathbb{R}^{n}$.

d. $\omega(x)$ es invariante respecto de $\dot{x}=f(x)$.

e. Si $x$ es un punto de descanso $\omega(x)=\{x\}$.

f. $\omega(x)$ es compacto y también arcoconexo.

Teorema 3.1 (Lyapunov) Sea $\dot{x}=f(x)$ un sistema autónomo definido en algún abierto $G$ en $\mathbb{R}^{n}$. Sea $V: G \rightarrow \mathbb{R}$ una función continuamente diferenciable. Si $x(t)$ es una solución de $\dot{x}=f(x)$ tal que la derivada de la función $F(t)=V(x(t))$ es $\geq 0(\leq 0)$. Entonces

$$
\begin{gathered}
\omega(x) \cap G \subseteq\{x \in G / \nabla V(x) \cdot f(x)=0\} \\
(\alpha(x) \cap G \subseteq\{x \in G / \nabla V(x) \cdot f(x)=0\})
\end{gathered}
$$

Definición 3.3 Sea $V: \operatorname{int}\left(\mathbb{R}_{+}^{2}\right) \rightarrow \mathbb{R}$ tal que $V(p, q)=e H(p)+c G(q)$ donde $H(p)=\bar{p} \ln p-p$ y $G(q)=\bar{q} \ln q-q$.

Nota 3.3 La función $V(p, q)$ tiene un máximo absoluto estricto en $(\bar{p}, \bar{q})$. 


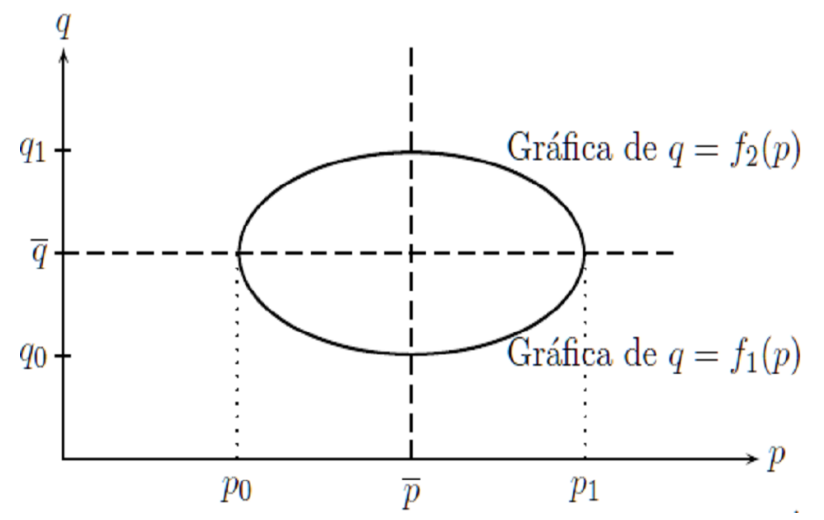

Figura 3: Curva $V(p, q)=k$.

Estudiaremos la curva $V(p, q)=k \operatorname{con} k<V(\bar{p}, \bar{q})$. Existen $p_{0}, p_{1}, q_{0}, q_{1}$ únicos tales que $0<p_{0}<\bar{p}<p_{1}, 0<q_{0}<\bar{q}<q_{1}, V\left(p_{0}, \bar{q}\right)=V\left(p_{1}, \bar{q}\right)=$ $V\left(\bar{p}, q_{0}\right)=V\left(\bar{p}, q_{1}\right)=k$ y $V(p, q)=k$ implica $p_{0} \leq p \leq p_{1}, q_{0} \leq q \leq q_{1}$.

La curva $V(p, q)=k$ está determinada por la gráfica de dos funciones $f_{1}(p), f_{2}(p)$ con $p \in\left[p_{0}, p_{1}\right]$ tales que $f_{1}(p)<f_{2}(p)$ si $\left.p \in\right] p_{0}, p_{1}\left[, f_{1}\left(p_{0}\right)=\right.$ $f_{2}\left(p_{0}\right)=f_{1}\left(p_{1}\right)=f_{2}\left(p_{1}\right)=\bar{q}, f_{1}$ es estrictamente creciente en $\left[\bar{p}, p_{1}\right]$ y es estrictamente decreciente en $\left[p_{0}, \bar{p}\right]$ y cóncava hacia arriba en $\left[p_{0}, p_{1}\right] ; f_{2}$ es estrictamente creciente en $\left[p_{0}, \bar{p}\right]$ y estrictamente decreciente en $\left[\bar{p}, p_{1}\right]$ y cóncava hacia abajo en $\left[p_{0}, p_{1}\right]$.

En resumen la gráfica de la curva $V(p, q)=k$ tiene la forma mostrada en la Figura 3.

Proposición 3.1 Sea $k$ tal que $k<V(\bar{p}, \bar{q})$, entonces tenemos que $V\left(p^{\prime}, q^{\prime}\right)>k$ si y solo si $\left(p^{\prime}, q^{\prime}\right)$ pertenece al interior de la curva $V(p, q)=k$.

Esta proposición está demostrada en [2].

Observación 4.2 Sea $(p(t), q(t)), t \in] a, b[$ es una solución de la ecuación diferencial (19) y ] $a, b[$ es el intervalo máximo de definición de $(p(t), q(t))$. Si $\left\{(p(t), q(t)) / t \geq t^{\prime}, t \in\right] a, b[\}$ está contenido en un compacto para algún $\left.t^{\prime} \in\right] a, b[$ entonces $b=+\infty$ y en particular la órbita de $(p(t), q(t)), t \in] a, b[$ contiene al conjunto $\left\{(p(t), q(t)) / t \geq t^{\prime}\right\}$.

Probemos esta observación. Suponga por contradicción que $b \in \mathbb{R}$; como el conjunto $\left\{(p(t), q(t)) / t \geq t^{\prime}, t \in\right] a, b[\}$ está contenido en un conjunto 
compacto, entonces existe $k>0$ tal que $\left.|p(t)|,|q(t)| \leq k, \forall t \geq t^{\prime}, t \in\right] a, b[$. Entonces

$$
\begin{gathered}
|\dot{p}|=|(-a-b p+c q) p| \leq(a+b|p|+c|q|)|p| \leq(a+b k+c k) k \quad \text { y } \\
|\dot{q}|=|(w-e p) q| \leq(w+e|p|)|q| \leq(w+e k) k
\end{gathered}
$$

si $k_{1}=(a+b k+c k) k, k_{2}=(w+e k) k$ entonces $|\dot{p}| \leq k_{1},|\dot{q}| \leq k_{2}, \forall t \geq t^{\prime}$, $t \in] a, b[$.

Si $\left.t_{1}, t_{2} \geq t^{\prime}, t_{1}, t_{2} \in\right] a, b[$ por el teorema del valor medio se tiene que

$$
\left|p\left(t_{1}\right)-p\left(t_{2}\right)\right|=\left|\dot{p}\left(t^{*}\right)\right|\left|t_{1}-t_{2}\right| \leq k_{1}\left|t_{1}-t_{2}\right| \quad\left(t^{*} \text { entre } t_{1}, t_{2}\right) ;
$$

por lo tanto $\left|p\left(t_{1}\right)-p\left(t_{2}\right)\right| \leq k_{1}\left|t_{1}-t_{2}\right|, \forall t_{1}, t_{2} \geq t^{\prime}$ y $\left.t_{1}, t_{2} \in\right] a, b[$. Similarmente se tiene que $\left|q\left(t_{1}\right)-q\left(t_{2}\right)\right| \leq k_{2}\left|t_{1}-t_{2}\right|, \forall t_{1}, t_{2} \geq t^{\prime}$ y $\left.t_{1}, t_{2} \in\right] a, b[$.

Sea $\left(t_{k}\right)$ cualquier secuencia de $] a, b\left[\right.$ tal que $\forall k, t_{k} \geq t^{\prime}$ y $\lim _{k \rightarrow+\infty} t_{k}=b$; entonces se tiene que $\left|p\left(t_{k}\right)-p\left(t_{k^{\prime}}\right)\right| \leq k_{1}\left|t_{k}-t_{k^{\prime}}\right|$, por lo tanto $\left(p\left(t_{k}\right)\right)$ es una secuencia de Cauchy y así $\lim _{k \rightarrow+\infty} p\left(t_{k}\right)$ existe y es real. Si $\left(t_{k}\right)$, $\left(t_{k}^{\prime}\right)$ son secuencias de $] a, b\left[\right.$ tales que $t_{k}, t_{k}^{\prime} \geq t^{\prime}, \forall k$ y $t_{k}, t_{k}^{\prime} \rightarrow b$ si $k \rightarrow$ $+\infty$; sabemos que $\lim _{k \rightarrow+\infty} p\left(t_{k}\right), \lim _{k \rightarrow+\infty} p\left(t_{k}^{\prime}\right)$ existen y son reales y como $\left|p\left(t_{k}\right)-p\left(t_{k}^{\prime}\right)\right| \leq k_{1}\left|t_{k}-t_{k}^{\prime}\right|$ y $k_{1}\left|t_{k}-t_{k}^{\prime}\right| \rightarrow 0$, si $k \rightarrow+\infty$, entonces $\lim _{k \rightarrow+\infty} p\left(t_{k}\right)=\lim _{k \rightarrow+\infty} p\left(t_{k}^{\prime}\right)$. Esto demuestra que $\lim _{t \rightarrow b^{+}} p(t)$ existe y es real. Similarmente se prueba que $\lim _{t \rightarrow b^{+}} q(t)$ existe y es real. Por el teorema de continuación de soluciones de ecuaciones diferenciales existe $b^{\prime}>b, b^{\prime} \in \mathbb{R}$ tal que $(p(t), q(t)), t \in] a, b^{\prime}[$ es solución de la ecuación diferencial (19). Pero esto contradice la maximalidad de $] a, b[$; por lo tanto $b=+\infty$.

Teorema 3.2 Si $(p(t), q(t))$ con $t \in I$ intervalo abierto máximo, $0 \in I$ es una solución de la ecuación diferencial

$$
\left\{\begin{array}{c}
\dot{p}=(-a-b p+c q) p \\
\dot{q}=(w-e p) q
\end{array}\right.
$$

con $a, b, c, w, e>0$ en $\operatorname{int}\left(\mathbb{R}_{+}^{2}\right)$, tal que $(p(0), q(0)) \neq(\bar{p}, \bar{q})$. Entonces la órbita de esa solución contiene al conjunto $\{(p(t), q(t)) / t \geq 0\}$ y este conjunto es una espiral que gira alrededor del punto $(\bar{p}, \bar{q})$ en el sentido de las manecillas del reloj $y$

$$
\lim _{t \rightarrow+\infty}(p(t), q(t))=(\bar{p}, \bar{q}) .
$$


Prueba. Probamos primero que si $t_{2}>t_{1}$, entonces $\left(p\left(t_{2}\right), q\left(t_{2}\right)\right)$ está en el interior de la curva $V(p, q)=k$, donde $k=V\left(p\left(t_{1}\right), q\left(t_{1}\right)\right)$. Tenemos que:

$$
\begin{gathered}
\frac{d}{d t}(V(p(t), q(t)))=\frac{\partial V}{\partial p} \cdot \dot{p}(t)+\frac{\partial V}{\partial q} \cdot \dot{q}(t) \\
=e\left(\frac{\bar{p}}{p}-1\right) p(-a-b p+c p)+c\left(\frac{\bar{q}}{q}-1\right) q(w-e p)
\end{gathered}
$$

$(\operatorname{como} a=-b \bar{p}+c \bar{q}, w=e \bar{p})$

$$
\begin{gathered}
=e\left(\frac{\bar{p}}{p}-1\right) p(b \bar{p}-c \bar{q}-b p+c q)+c\left(\frac{\bar{q}}{q}-1\right) q(e \bar{p}-e p) \\
=e(\bar{p}-p)(b(\bar{p}-p)-c(\bar{q}-q))+c(\bar{q}-q) e(\bar{p}-p) \\
=e(\bar{p}-p)(b(\bar{p}-p)-c(\bar{q}-q)+c(\bar{q}-q)) \\
=e b(\bar{p}-p)^{2} \geq 0 .
\end{gathered}
$$

Probemos que existe $\tilde{t} \in] t_{1}, t_{2}[$ tal que $p(\tilde{t}) \neq \bar{p}$. Suponga por contradicción que $\forall t \in] t_{1}, t_{2}[, p(t)=\bar{p}$, entonces en $] t_{1}, t_{2}[, \dot{p}=0$, lo que implica que $-a-$ $b \bar{p}+c q=0$ en $] t_{1}, t_{2}[$ y entonces $(p(t), q(t))=(\bar{p}, \bar{q}) \forall t$ y así $(p(0), q(0))=$ $(\bar{p}, \bar{q})$, lo cual es una contradicción. Por lo tanto, existe $\tilde{t} \in] t_{1}, t_{2}[$ tal que $p(\tilde{t}) \neq \bar{p}$. Escogemos un intervalo centrado en $\tilde{t}$ contenido en $] t_{1}, t_{2}[$ tal que en tal intervalo $p(\tilde{t}) \neq \bar{p}, \forall t$ en tal intervalo. Sean $\tilde{t}_{1}, \tilde{t}_{2}$ en ese intervalo tal que $\tilde{t}_{1}<\tilde{t}_{2}$, entonces

$$
\frac{d}{d t}(V(p(t), q(t)))>0 \quad \forall t \in\left[\tilde{t}_{1}, \tilde{t}_{2}\right]
$$

por lo tanto $V(p(t), q(t))$ es estrictamente creciente en $\left[\tilde{t}_{1}, \tilde{t}_{2}\right]$ y como $t_{1}<$ $\tilde{t}_{1}<\tilde{t}_{2}<t_{2}$ se tiene:

$$
V\left(p\left(t_{1}\right), q\left(t_{1}\right)\right) \leq V\left(p\left(\tilde{t_{1}}\right), q\left(\tilde{t}_{1}\right)\right)<V\left(p\left(\tilde{p}_{2}\right), q\left(\tilde{t}_{2}\right)\right) \leq V\left(p\left(t_{2}\right), q\left(t_{2}\right)\right)
$$

y así se tiene que $V\left(p\left(t_{1}\right), q\left(t_{1}\right)\right)<V\left(p\left(t_{2}\right), q\left(t_{2}\right)\right)$ y por lo tanto el punto $\left(p\left(t_{2}\right), q\left(t_{2}\right)\right)$ es un punto interior de la curva $V(p, q)=k$, donde $k=$ $V\left(p\left(t_{1}\right), q\left(t_{1}\right)\right)$.

En particular se tiene que

$$
\left\{\left(p\left(t_{1}\right), q\left(t_{1}\right)\right) / t \geq 0, t \in I\right\} \subseteq\{(p, q) / V(p, q) \geq V(p(0), q(0))\},
$$

Pero el conjunto $\{(p, q) / V(p, q) \geq k\}$ es compacto $\forall k$. Entonces esto implica que por la observación anterior al teorema, la órbita de la solución 
$(p(t), q(t))$ contiene al conjunto $\{(p(t), q(t)) / t \geq 0\}$. También tenemos que

$$
\omega(p(0), q(0)) \subseteq\{(p, q) / V(p, q) \geq V(p(0), q(0))\},
$$

en particular $\omega(p(0), q(0)) \subseteq \operatorname{int}\left(\mathbb{R}_{+}^{2}\right)$ y $\omega(p(0), q(0)) \neq \emptyset$.

Por otro lado, como $\frac{d}{d t}(V(p(t), q(t))) \geq 0 \forall t$, podemos aplicar el teorema de Lyapunov y entonces

$$
\omega(p(0), q(0)) \subseteq\left\{(p, q) \in \operatorname{int}\left(\mathbb{R}_{+}^{2}\right) / \nabla V(p, q) \cdot f(p, q)=0\right\}
$$

donde $f(p, q)=((-a-b p+c q) p,(w-e p) q)$.

$$
\begin{gathered}
\text { Pero } \nabla V(p, q)=\left(e\left(\frac{\bar{p}}{p}-1\right), c\left(\frac{\bar{q}}{q}-1\right)\right) \text { y entonces } \\
\begin{aligned}
\nabla V(p, q) \cdot f(p, q)=e\left(\frac{\bar{p}}{p}-1\right) p(-a-b p+c q)+c\left(\frac{\bar{q}}{q}-1\right) q(w-e p) \\
=e b(\bar{p}-p)^{2} .
\end{aligned}
\end{gathered}
$$

Entonces $\nabla V(p, q) \cdot f(p, q)=0$ si y solo si $p=\bar{p}$. Por lo tanto $\omega(p(0), q(0)) \subseteq\left\{(p, q) \in \operatorname{int}\left(\mathbb{R}_{+}^{2}\right) / p=\bar{p}, q>0\right\}$. Pero sabemos que $\omega(p(0), q(0)) \neq \emptyset$ y como $\omega(p(0), q(0))$ es invariante, si $\left(\tilde{p}_{0}, \tilde{q}_{0}\right)$ pertenece a $\omega(p(0), q(0))$ implica que $\tilde{p_{0}}=\bar{p}$. Sea $(\tilde{p}(t), \tilde{q}(t))$ una solución de la ecuación diferencial tal que $(\tilde{p}(0), \tilde{q}(0))=\left(\tilde{p}_{0}, \tilde{q}_{0}\right)$. Como $\omega(p(0), q(0))$ es invariante implica $(\tilde{p}(t), \tilde{q}(t)) \in \omega(p(0, q(0))) \forall t$, entonces $\tilde{p}(t)=\bar{p} \forall t \Rightarrow$ $\dot{\tilde{p}}=0$ y como $\tilde{q}(t)>0 \forall t$ se tiene que $-a-b \bar{p}+c \tilde{q}(t)=0 \quad \forall t$ lo que implica que $\tilde{q}(t)=\bar{q} \forall t$ y así se tiene que $\tilde{q}(0)=\bar{q}$ y entonces $\left(\tilde{p}_{0}, \tilde{q}_{0}\right)=(\bar{p}, \bar{q})$. Por lo tanto $\omega(p(0), q(0)) \subseteq\{(\bar{p}, \bar{q})\}$ y como $\omega(p(0), q(0)) \neq \emptyset$ se tiene que $\omega(p(0), q(0))=\{(\bar{p}, \bar{q})\}$ y entonces

$$
\lim _{t \rightarrow+\infty}(p(t), q(t))=(\bar{p}, \bar{q})
$$

Por lo tanto hemos probado que si $t_{2}>t_{1}$ entonces $\left(p\left(t_{2}\right), q\left(t_{2}\right)\right)$ está en el interior de la curva $V(p, q)=k$ con $k=V\left(p\left(t_{1}\right), q\left(t_{1}\right)\right)$ y $\lim _{t \rightarrow+\infty}(p(t), q(t))=(\bar{p}, \bar{q})$; entonces el conjunto $\{(p(t), q(t)) / t \geq 0\}$ es una espiral que gira alrededor de $(\bar{p}, \bar{q})$ en el sentido de las manecillas del reloj y que converge al punto $(\bar{p}, \bar{q})$, completándose esto la prueba del teorema.

Nota 3.4 Podemos dar una representación gráfica de la semiórbita de la ecuación diferencial del teorema anterior (ver Figura 4). 


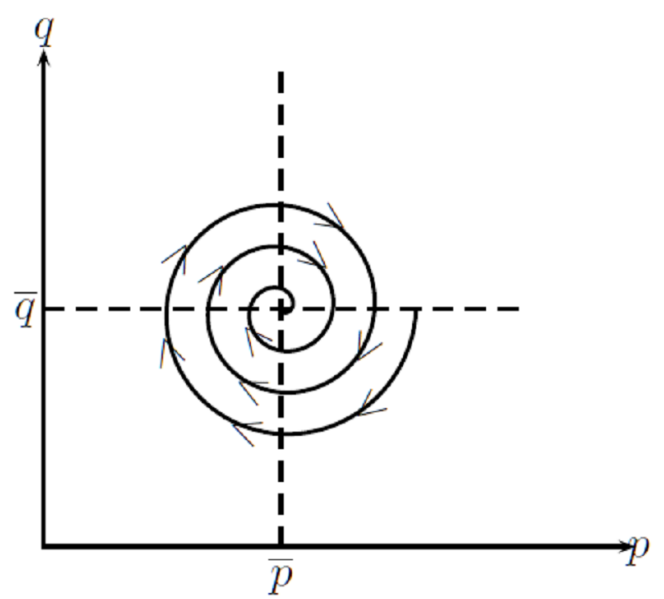

Figura 4: Semiórbita de la ecuación diferencial del teorema 3.2.

\section{Bibliografía}

[1] Acuña, O.; Ulate, F. (1994) Matrices No Negativas. Editorial de Universidad de Costa Rica.

[2] Acuña, O.; Ulate, F. (2008) "Adam Smith y sistemas dinámicos", Revista Ciencias Económicas 26(1): 171-185.

[3] Ascanio, A. (2004) "Teoría y práctica: curva de la demanda del petróleo crudo venezolano", Universidad Simón Bolívar, Caracas.

[4] Barrel, R.; Pomerantz, O. (2004) "Oil prices and the world economy", National Institute of Economic and Social Research, London.

[5] Campbell, C.J. (2002) "Forecasting global oil supply 2000-2050", M. King Hubbert Center for Petroleum Supply Stydies, USA.

[6] Coderch, M. (2006) "El fin del petróleo barato", Foreign Policy, Edición Española, .

[7] De Santis, R. (2000) "Crude oil price fluctuations and Saudi Arabian behaviour", Kiel Working Paper No. 1014. Kiel Institute of World Economics, Alemania.

[8] Economagic.com (2006) "Economic Time Series Page. Price of West Texas Intermediate Crude". Monthly NSA, Dollar Per 
Barrel.

http://www.economagic.com/emcgi/data.exe/var/west-texascrude-long

[9] Financial Forecast Center Home Page.

http://www.forecast.org/National

[10] Gibbons, R. (1992) Game Theory for Applied Economists. Princeton University Press.

[11] Hallan, T.G.; Levin, S.A. (1986) Mathematical Ecology: An Introduction. Biomathematics 17, Springer, Berlin.

[12] Hirsch, M.W. (1982) "Systems of differential equations which are competitive or cooperative: I Limit sets", SIAM J. Math. Anal. 13: $167-179$.

[13] Hofbauer, J.; Sigmund, K. (1998) Evolutionary Games and Populations Dynamics. Cambridge University Press, Cambridge U.K.

[14] Lotka, A.J. (1957) Elements of Mathematical Biology. Dover, New York.

[15] OPEP (2005) Boletín Anual Estadístico, www.opec.org

[16] Perko, L. (2001) Differential Equations and Dynamical Systems. Springer-Verlag, Berlin.

[17] Pindyck, R. (2005) The Long-Run Evolution of Energy Prices. Massachusetts Institute of Technology. Cambridge.

[18] Rotemberg, J.; Garth, S. (1985) "A supergame theoretic model of price wars during boorns", American Economic Review 78: 390407.

[19] Ulate, F. (2006) "Formalización de una teoría de la mentalidad", Revista de Matemática: TEoría y Aplicaciones 13(1): 53-80.

[20] Vegan Society. http://www.vegansociety.com/ 